\title{
Modest weight loss improves leptin to adiponectin ratio and induces insulin and leptin resensitivization in individuals with obesity.
}

\section{Victoria Therese Isaksen ( $\nabla$ dr.isaksen@gmail.com )}

UiT The Arctic University of North Norway https://orcid.org/0000-0003-2121-0755

\section{Maria Arlen Larsen}

UiT The Arctic University of Norway: UiT Norges arktiske universitet

\section{Rasmus Goll}

UiT The Arctic University of Norway: UiT Norges arktiske universitet

\section{Eyvind J. Paulssen}

UiT The Arctic University of Norway: UiT Norges arktiske universitet Jon Ragnar Florholmen

UiT The Arctic University of Norway: UiT Norges arktiske universitet

\section{Research}

Keywords: Insulin resistance, Leptin resistance, Leptin to adiponectin ratio, Leptin, Adiponectin, Metabolic syndrome, Obesity, Postprandial triglycerides, Weight loss

Posted Date: October 1st, 2021

DOl: https://doi.org/10.21203/rs.3.rs-936579/v1

License: (c) (1) This work is licensed under a Creative Commons Attribution 4.0 International License. Read Full License 


\section{Abstract}

Background Weight loss is important to reduce the risk of metabolic complications in obese individuals, in whom dysregulated adipokines play a central role. This study aims to investigate whether dysregulated adipokines and postprandial triglycerides (TG) improve with a modest weight loss. Methods Individuals with obesity were recruited among patients at the University Hospital of North Norway and the Stamina Health weight loss rehabilitation program. We measured resting energy expenditure (REE), and calculated the Homeostasis Model Assessment of Insulin Resistance (HOMA-IR), leptin to adiponectin (L:A) ratio, indirect leptin sensitivity (REE:leptin ratio), postprandial TG clearance at $6 \mathrm{~h}$, and TG response before and after weight loss. The goal of the weight loss intervention was a loss of $\geq 5 \%$ of initial total body weight. Results Of the 28 participants who attended two scheduled assessments, 13 lost $\geq 5 \%$ body weight. HOMA-IR (-23.1\%), REE:leptin ratio (+80.1\%) and L:A ratio (-45.7\%) significantly improved with weight loss, whereas there was no improvement of postprandial TG response or clearance. No significant changes were observed in the non-weight loss group. Conclusion Metabolic dysregulation, as insulin and leptin resistance, but not postprandial TG improve with a modest weight loss in individuals with obesity.

\section{Background}

Overweight and obesity are important risk factors for morbidity and mortality $(1,2)$, mainly because of their association with metabolic dysfunction and increased risk of cardiovascular disease (CVD), cancer, and type 2 diabetes mellitus (T2DM) $(1,3)$.

Dyslipidaemia is an essential part of the metabolic syndrome, in addition to abdominal obesity and insulin resistance (4). We have previously demonstrated that the postprandial triglyceride response (TGR) is altered in healthy individuals with obesity, with a delayed postprandial triglyceride (TG) clearance compared to healthy, normal weight individuals (5). Because humans spend most of their waking hours in the postprandial state, this increased period of triglyceride exposure in obese individuals contributes to an increased risk of both CVD and T2DM, by increasing atherosclerosis and induce insulin resistance (6, 7).

The adipokines leptin and adiponectin are, together with free fatty acids (FFA), critical mediators in adipocytes to maintain metabolic homeostasis (8-10). Moreover, adipokines play a central role in modulating inflammation $(11,12)$, another contributing mechanism to CVD. In individuals with overweight and obesity, alterations of adipokine levels and their function are essential factors of the pathophysiologic mechanisms causing the metabolic syndrome, CVD, T2DM and other complications to obesity $(12,13)$.

Obesity is related to leptin resistance, a well-known concept first described in 2000 (14). Leptin levels are correlated to fat mass and thus signals energy balance (16). Obese individuals are almost always hyperleptinaemic while having a normal resting energy expenditure (REE), whereas normal weight individuals maintain a normal REE on lower leptin levels (17). Reduced leptin levels signal energy 
deficiency, which in turn induces counter-regulatory responses, of which reduced REE is one (16). Thus, the effect of leptin on REE seems to be feasible as an indirect marker of leptin sensitivity, by using the REE to leptin (REE:leptin) ratio (17).

Leptin and adiponectin changes are seen after a fatty meal in normal weight individuals, but not in obese individuals (18). Furthermore, the leptin to adiponectin ( $L: A)$ ratio has been proven to correlate well with other aspects of metabolic dysregulation and risk of chronic metabolic disease $(19,20)$. Individuals with obesity and elevated L:A ratio tend to have a delayed TG clearance, as well as both insulin and leptin resistance, making the L:A ratio a useful surrogate marker for the metabolic syndrome (18).

A modest weight loss of $\geq 5 \%$ improves metabolic disturbances and clinical features of the metabolic syndrome and complications of T2DM, with the improvement of insulin sensitivity being a crucial element $(21,22)$. Furthermore, because of compensatory metabolic mechanisms during weight loss, a modest weight loss might be easier to maintain in the long run than a $>10 \%$ weight loss (23). Improvement of central leptin satiety signalling, as expressed in feeding behaviour, with weight loss and reduced leptin levels have previously been demonstrated (15). However, to our knowledge, documentation of leptin resensitization has only been described in experimental animal weight loss models, not in humans (15).

Thus, this study aimed to investigate if a modest weight loss of $\geq 5 \%$ is sufficient for significant improvement of the L:A ratio over the cut-off values previously identified (18), and if a corresponding improvement of other biomarkers of subclinical metabolic dysregulation occurs $(18,24)$.

\section{Materials And Methods}

We recruited participants from the Centre for Obesity, Department of Gastroenterology and Nutrition, from the obesity rehabilitation program at Stamina Health Troms $\emptyset$ and by posters placed at the Department of Clinical Nutrition and Department of Endocrinology at the University Hospital of North Norway (UNN). Eligible patients were provided with oral and written information and signed a written consent to participate.

Inclusion of participants is shown in Figure 1.

Inclusion criteria for the study population were a baseline body mass index (BMI) $\geq 30 \mathrm{~kg} / \mathrm{m}^{2}$ and age $\geq 18$ years. Exclusion criteria were smoking, pregnancy, severe mental illness, previous heart disease, medically treated diabetes mellitus and kidney failure. We excluded patients who, for any reason, dropped out of the weight loss program. Height, body weight, blood pressure and pulse were measured. Total, abdominal and gynoid fat percentage, total fat mass $(\mathrm{kg})$ and total muscle mass $(\mathrm{kg})$ were obtained from body composition measured at baseline and follow-up after weight loss treatment, using Dual-Energy Xray Absorptiometry (DEXA; Lunar Prodigy Advance, GE Health Care, USA). 
For our control group we recruited 17 healthy, normal weight participants from the general population. Inclusion criteria were BMI in normal range, between 18 and 40 years of age, normotensive, normoglycaemic, normolipaemic and no history of diabetes. Exclusion criteria were otherwise the same as for our obese participants. The control participants underwent the same measurements and tests as our obese participants. Their results were used to create $95 \%$ confidence intervals $(\mathrm{Cl})$ for normal values in our metabolic parameters.

\section{Weight loss intervention}

The weight loss goal for the participants with obesity was a minimum of $\geq 5 \%$ of the baseline body weight, as this amount of weight loss is accepted as clinically meaningful (24). The post-weight loss tests were performed within two years after the weight loss intervention. A shortened follow-up period was selected for participants who lost weight quickly, in order to avoid loss to follow-up due to weight regain.

Weight loss intervention was performed either individually or in treatment groups in the programs from where participants were recruited. The intervention was based on Norwegian national guidelines for diet and exercise $(25,26)$. Since the study aimed to investigate the effects of weight loss per se, the instructions for weight loss methods were kept liberal and not formally recorded. Participants were free to adjust their diet and exercise according to their own preferences, within national guidelines.

All 50 participants who underwent baseline tests were offered to undergo the second round of tests regardless of the amount of weight loss during follow-up. 22 out of 37 participants in the non-weight loss group declined the offer and was hence lost to follow-up, while 15 accepted. All 13 participants in the weight loss group accepted the offer.

\section{Insulin sensitivity}

We performed a $2 \mathrm{~h}$ Oral Glucose Tolerance Test (OGTT) (18). Participants had their regular diet and abstained from vigorous exercise three days before the test and showed up at 08:00 am after $12 \mathrm{~h}$ of overnight fasting. The test was conducted by oral intake of $75 \mathrm{~g}$ glucose dissolved in water (27). We collected blood samples in both the fasting state and 30, 60, 90 and 120 minutes after glucose intake, in which serum (s-) glucose and s-insulin were measured using ELISA kits (DRG Insulin Elisa kit, DRG Instruments $\mathrm{GmbH}$, Germany) (5). We determined insulin sensitivity by calculation of the HOMA-IR (28), as it has previously proven to correspond well to the whole body insulin sensitivity index (WBISI) $(27,29)$.

\section{S-leptin and adiponectin measurements}

Both leptin and free adiponectin were analysed from frozen serum drawn at all sample times, both during OFTT and OGTT, using ELISA kits (DRG Diagnostics, Marburg, Germany) for leptin (sandwich ref. EIA2395) and adiponectin (human, ref. EIA-4574), respectively. From these measurements, the L:A ratio was calculated as follows: 


\section{$L:$ A ratio $=\frac{\text { serum leptin }}{\text { serum adiponectin }}$}

As the intra individual variation was minimal between OFTT and OGTT measurements, OFTT values were selected for the statistical analyses.

Leptin sensitivity

Leptin sensitivity was calculated as the ratio of Resting Energy Expenditure (REE) to fasting s-leptin (17, 30). We performed REE measurements by a canopy test with an indirect calorimetry device from Medical Graphics CPX metabolic cart (St Paul, MN, USA). The test protocol is previously described (18). After the completion of REE measurement, the OGTT was performed (17).

\section{Postprandial triglyceride clearance}

To measure postprandial TGR and TG clearance, we performed an Oral Fat Tolerance Test (OFTT) on a separate day from the OGTT $(31,32)$. Preparations for the OFTT were the same as for the OGTT. Fasting blood samples were drawn, and a meal of sour cream porridge was served, containing $1 \mathrm{~g}$ of fat per $\mathrm{kg}$ of body weight (32). The participants ingested the meal within 30 minutes, and blood samples were drawn from the antecubital vein in a seated position at baseline and 2, 4, 6 and $8 \mathrm{~h}$ postprandially.

The Department for Clinical Biochemistry at UNN analyzed fasting serum samples for TG, total cholesterol, low density lipoprotein (LDL) cholesterol and high-density lipoprotein (HDL) cholesterol. All samples were analysed using the same methods, equipment and reference values as the study by Larsen et al. (5).

The TGR was defined as the average of the two highest postprandial TG concentrations, minus the baseline concentration $(5,32)$.

The formula for calculating TG clearances (32) at time X was as follows:

$$
\text { Clearance } X h=100 \times\left(1-\frac{T G_{X}-T G_{0 h}}{T G_{\max }-T G_{0 h}}\right)
$$

Cut-off values for metabolic parameters

Normalization of metabolic parameters were defined as reaching the $95 \% \mathrm{Cl}$ of our healthy, normal weight control group. The cut-off values were as follows: TG clearance at $6 \mathrm{~h} \geq 88 \%$, TGR $\leq .67, \mathrm{HOMA}$ IR $\leq 1.83$, WBISI $\geq 131.4$, L:A ratio $\leq 1.19$ and REE:leptin ratio $\geq 114.5$ (18). 
For statistical calculations, we used IBM SPSS Statistics 25 for Windows (SPSS Inc., Chicago, IL, USA). For calculating TG clearance and postprandial TGR, we used Microsoft Excel (Microsoft corp., Redmond, WA, USA). Plots were generated in GraphPad Prism 7 (GraphPad Software, La Jolla, CA, USA).

We used parametric tests on raw or transformed variables that resembled a normal distribution visually or by skewness/kurtosis. Otherwise, non-parametric tests were performed.

\section{Results}

\section{Baseline characteristics}

We included 28 Caucasian participants in this study with a weight loss of 0-30\%. Seven participants $(25 \%)$ were male and 21 (75\%) were female. Among these, 15 participants $(54 \%)$ lost $<5 \%$ body weight whereas 13 participants ( $46 \%$ ) lost $\geq 5 \%$ body weight, including 5 participants (18\%) who lost $\geq 10 \%$. Median weight loss in the weight loss group was $10.0 \mathrm{~kg}$.

Clinical, anthropometric, and metabolic characteristics are shown in Table 1. There were no significant differences in baseline characteristics between the weight loss and non-weight loss group, with the exception of REE $(p=0.006), T G(p=0.011)$ and HDL-cholesterol $(p=0.004)$ levels. For leptin and adiponectin measurements, only data from OFTT samples are shown.

Four participants (three in the weight loss group, one in the non-weight loss group) used antihypertensive medication at baseline, two used thyroid replacement drugs (one in each group) and three used lipid lowering drugs (three in the weight loss and one in the non-weight loss group). Two participants in the weight loss group had hypertension at baseline. All female participants except one had LDL-cholesterol levels $<4.3 \mathrm{mmol} / \mathrm{L}$, the upper limit of normal for females $<50$ years. All male participants except one had LDL-cholesterol levels $<4.7 \mathrm{mmol} / \mathrm{L}$, the upper limit of normal for males $<50$ years.

Our normal weight controls had a median fasting s-leptin of $8.5 \mathrm{ng} / \mathrm{mL}$ and fasting s-adiponectin of 11.8 $\mu \mathrm{g} / \mathrm{mL}$.

\section{L:A ratio}

There were significant improvements between pre- and post-intervention visits for fasting s-leptin and L:A ratio for the weight loss group, but not for the non- weight loss group (Table 2). Participants with weight loss had a $45.7 \%(p=0.002)$ improvement in L:A ratio (Table 2$)$.

Figure 2 shows the case-by-case change in central variables before and after weight loss intervention. Most notably is the significant improvement in L:A ratio in participants in the weight loss group. The improvement was even greater in the subgroup of $\geq 10 \%$ weight loss, but not reaching the level of normality (cut-off value $\geq 1.88, p=0.030$, figure 2 ). In the non-weight loss group no significant changes were observed $(p=0.020)$. 
The REE:leptin ratio improved with $80.1 \%(p=0.005)$ in the weight loss group (table 2$)$ but not in the nonweight loss group. Furthermore, there were a tendency of greater improvements in leptin sensitivity in the subgroup of $\geq 10 \%$ weight loss, compared to $5-10 \%$ weight loss. Four of the $13(31 \%)$ participants in the subgroup achieved normalized leptin sensitivity after weight loss (REE:leptin ratio $\geq 114.5$, figure 2 ). No significant changes were observed in the non-weight loss group $(p=0.013)$.

\section{Insulin sensitivity}

Insulin sensitivity measured by HOMA-IR improved with $23.1 \%(p=0.011)$ in the weight loss group, where nine out of the $13(69 \%)$ participants had normal HOMA-IR values after weight loss (HOMA-IR $\leq 1.83$ ) Of these, five participants improved from insulin resistance at baseline.

There also was a significant improvement of $49.9 \%$ in WBISI in the weight loss group $(p=0.008)$. Two participants in this group normalized their WBISI, while one participant maintained a normal baseline WBISI. There also was a significant difference in WBISI delta values between weight loss and non-weight loss group ( $p=0.013$, Mann-Whitney $U$ test).

No significant changes were observed in the non-weight loss group for neither HOMA-IR nor WBISI (Table 2, figure 2).

\section{Improvement of postprandial triglycerides}

No significant differences in postprandial TG clearance at $6 \mathrm{~h}$ were seen in any of the groups $(6.6 \%, p=$ 0.807). (Table 2, figure 2).

\section{Discussion}

In this study we have examined the L:A ratio, indirect leptin sensitivity, insulin sensitivity, and postprandial TG metabolism in obese participants before and after a modest weight loss of $\geq 5 \%$. We found significant improvements in L:A ratio (-45.7\%), leptin sensitivity (-80.1\%) and insulin sensitivity (-23.1\%) in participants who acheived weight loss, compared to participants with no weight loss. Furthermore, our study shows that as little as $\geq 5 \%$ weight loss improve adipokines, but not TG metabolism.

We have previously reported that the L:A ratio is a feasible surrogate biomarker for early detection of metabolic disturbances in obesity (19). Moreover, we have previously reported a potential postprandial regulatory role of adiponectin and leptin which is impaired in obesity (18). The L:A ratio has been demonstrated to improve after a weight loss of $5-10 \%$ by Ferreira et al. and Talaei et al, among others. $(33,34)$.

Our present findings demonstrate an improvement in L:A ratio of $-45.7 \%$ in a small group of 13 participants with weight loss. Such a large improvement in this small dataset has major clinical 
significance, and supports a realistic weight loss goal that is potentially easier to maintain long term (23). The improvement of the L:A ratio is important as it is known to correlate to low-grade inflammation and thus risk of CVD $(36,37)$.

Frühbeck et al. proposed adiponectin:leptin ratio cardiovascular risk category limits as follows: normal risk $\geq 1$, moderate risk $0.5-1$ and high risk $<0.5$ (37). Inversely, this translates to our L:A ratio category limits of $\leq 1,1-2$ and $>2$, respectively. According to these limits of risk, three participants with weight loss crossed from high to moderate cardiovascular risk after weight loss while one participant crossed from moderate to low risk and one from high to low risk. In total $38 \%$ of the partcipants reduced their risk category.

A study by $\mathrm{Bi}$ et al. suggests that leptin contributes significantly more to the variance in REE than what is explained by fat mass (30). Furthermore, Rosenbaum et al. demonstrated that leptin administration reversed the decrease in energy expenditure after weight loss (38). We found that REE:leptin ratio improved with a median of $80.1 \%$. In addition, approximately one third $(31 \%)$ of the participants in the weight loss group had their REE:leptin ratio normalized after weight loss (18). Although resensitivization of leptin on satiety signalling is not possible to measure clinically in humans, one can speculate whether this demonstration of indirect resensitivization of leptin suggest a normalization of central leptin sensitivity as well.

Insulin sensitivity, as measured by the HOMA-IR or WBISI, improved by $23.1 \%$ and $49.9 \%$, resepectively, after weight loss. Moreover, five out of the $13(39 \%)$ participants obtained normalized insulin sensitivity as defined by HOMA-IR, while two out of $13(13 \%)$ normalized it as defined by WBISI $(18,30)$. This is in agreement with most other reports (22). The improved insulin sensitivity observed together with an improved L:A ratio is also in agreement with previous reports, describing a correlation between the two variables (42). Our findings of a substantial percentage of normalized insulin sensitivity further support the conclusion that a $5 \%$ weight loss is clinically meaningful for reducing obesity complications.

There were no significant improvement of postprandial TG clearance after $\geq 5 \%$ weight loss. This could be explained by the small number of participants that lost $\geq 10 \%$ body weight. Magkos et al. reported that improvements of TG and FFA were observed first after $11 \%$ and $16 \%$ weight loss, respectively (21). Therefore, normalization of postprandial TG clearance will most likely be observed only after a substantial weight loss in obese individuals.

The strengths of the present study are the use of various methods, particularly adipokine levels, reflecting the patophysiological mechanisms behind metabolic disorders in obesity to make comprehensive explanations of the results obtained by a modest weight loss. In addition to this, the generalizability of the weight loss method used in the study is high, as we did not assign one specific diet or exercise plan to our participants. We also included participants from different sources with broad inclusion criteria. The study was performed in a clinical setting, which also makes the generalizability high. 
The main weakness of our study design is the dropout rate and small sample size that renders us unable to perform parametric tests and to perform more complex analyses (ANOVA, etc.). Due to the sample size we mainly used repeated measures analyses, thus reducing the variability in our samples, making our results more reliable under the given circumstances.

There is also a significant imbalance between men and women included in our study. This could affect our results, as body composition and hence leptin concentrations differ between the sexes (43). However, the gender differences in our study is in accordance with other studies of obesity, and also with literature on such differences in the population of patients seeking help for health problems in general (44).

In conclusion, a modest weight loss of $\geq 5 \%$ improves sensitive metabolic surrogate markers like L:A ratio, leptin sensitivity (REE:leptin ratio) and insulin sensitivity. However, markers of postprandial TG clearance did not improve at the present level of weight loss. Our results support that a realistic and achievable weight loss goal of $\geq 5 \%$ for obese individuals does reduce the risk of metabolic complications.

\section{Abbreviations}

ALT Alanine aminotransferase

ANOVA Analysis of variance

Apo $\quad$ Apolipoprotein (A1, B and E)

AST Aspartate aminotransferase

BMI Body mass index

BP Blood pressure

$\mathrm{Cl} \quad$ Confidence interval

CVD Cardiovascular disease

DEXA Dual-energy X-ray absorptiometry

ELISA Enzyme-linked immunosorbent assay

FFA Free fatty acids

Y-GT Gamma glutamyl transferase

h Hours

HDL High-density lipoprotein 
HOMA-IR Homeostasis model assessment of insulin resistance
L:A Leptin to adiponectin (ratio)
LDL Low-density lipoprotein

OGTT Oral glucose tolerance test

OFTT Oral fat tolerance test

REE Resting energy expenditure

REK Regional Etisk Komité (Regional Ethics Committee)

TG Triglyceride

TGR Triglyceride response

UiT University of Troms $\varnothing$

UNN University hospital of North Norway

WBISI Whole body insulin sensitivity index

\section{Declarations}

Availability of data and materials: The dataset supporting the conclusions of this article is available in the UiT Open Research Data repository at https://doi.org/10.18710/KRYLXN

Competing interests: The authors have nothing to disclose.

Funding: This study was funded by the Medical Students Research Programme at the Faculty of Health Sciences, UiT The Arctic University of Norway. The publication charges for this article have been funded by a grant from the publication fund of UiT The Arctic University of Norway

Ethics: All participants signed a written consent form. The Regional Ethics Committee of North Norway (2011/1677/REK Nord) approved the study. Consent for publication of results was not applicable, as no individual participant is identifiable in this paper.

Author's contributions: Authors VTI and MAL conducted participant inclusion and data collection. MAL performed weight loss follow-up for some of the participants. VTI and RG performed statistical analyses. JF was the principal investigator.

All authors participated in the planning of the study, the interpretation of results and the process of writing and editing the manuscript. 
Acknowledgements: The authors are sincerely grateful for the contributions to this study done by the staff at the Gastroenterological Laboratory, UNN. Clinical research nurse Odd Sverre Moen contributed significantly to participant examinations. Bioengineers Marian Remijn and Line Wilsgaard analysed adipokine samples and performed DEXA and REE tests. Centre for Obesity nurse Torunn Dreyer recruited study participants and carried out participant follow-up, including weight loss treatment. Clinical nutritionist Hugo Nilssen and the staff at Stamina Health, Troms $\varnothing$ also contributed significantly to weight loss follow-up.

\section{References}

1. O'Neill S, O'Driscoll L. Metabolic syndrome: a closer look at the growing epidemic and its associated pathologies. Obesity Reviews. 2015;16(1):1-12.

2. WHO. Obesity and overweight 2020 [updated 4/2020. Available from: http://www.who.int/mediacentre/factsheets/fs311/en/.

3. Rhee E-J. Being Metabolically Healthy, the Most Responsible Factor for Vascular Health. Diabetes Metab J. 2018;42(1):19-25.

4. Vekic J, Zeljkovic A, Stefanovic A, Jelic-Ivanovic Z, Spasojevic-Kalimanovska V. Obesity and dyslipidemia. Metabolism - Clinical and Experimental. 2019;92:71-81.

5. Larsen MA, Goll R, Lekhal S, Moen OS, Florholmen JR. Delayed clearance of triglyceride-rich lipoproteins in young, healthy obese subjects. Clinical Obesity. 2015;5(6):349-57.

6. Tenenbaum A, Klempfner R, Fisman EZ. Hypertriglyceridemia: a too long unfairly neglected major cardiovascular risk factor. Cardiovascular Diabetology. 2014;13:159.

7. Huet F, Roubille C, Roubille F. Is hypertriglyceridemia atherogenic? Current opinion in lipidology. 2019;30(4):291-9.

8. Stern JenniferH, Rutkowski JosephM, Scherer PhilippE. Adiponectin, Leptin, and Fatty Acids in the Maintenance of Metabolic Homeostasis through Adipose Tissue Crosstalk. Cell Metabolism. 2016;23(5):770-84.

9. Harris RBS. Direct and indirect effects of leptin on adipocyte metabolism. Biochimica et Biophysica Acta (BBA) - Molecular Basis of Disease. 2014;1842(3):414-23.

10. Wang ZV, Scherer PE. Adiponectin, the past two decades. Journal of Molecular Cell Biology. 2016;8(2):93-100.

11. Naylor C, Petri Jr WA. Leptin Regulation of Immune Responses. Trends in Molecular Medicine. 2016. 
12. Unamuno X, Gómez-Ambrosi J, Rodríguez A, Becerril S, Frühbeck G, Catalán V. Adipokine dysregulation and adipose tissue inflammation in human obesity. European Journal of Clinical Investigation. 2018;48(9):e12997.

13. Souza RA, Alves CMR, de Oliveira CSV, Reis AF, Carvalho AC. Circulating levels of adiponectin and extent of coronary artery disease in patients undergoing elective coronary angiography. Brazilian journal of medical and biological research = Revista brasileira de pesquisas medicas e biologicas. 2017;51(2):e6738.

14. Aizawa-Abe M, Ogawa Y, Masuzaki H, Ebihara K, Satoh N, Iwai H, et al. Pathophysiological role of leptin in obesity-related hypertension. The Journal of clinical investigation. 2000;105(9):1243-52.

15. Andreoli MF, Donato J, Cakir I, Perello M. Leptin resensitization: a reversion of leptin resistant states. J Endocrinol. 2019.

16. Elias CF, Purohit D. Leptin signaling and circuits in puberty and fertility. Cellular and Molecular Life Sciences. 2013;70(5):841-62.

17. Lustig RH, Sen S, Soberman JE, Velasquez-Mieyer PA. Obesity, leptin resistance, and the effects of insulin reduction. International journal of obesity and related metabolic disorders : journal of the International Association for the Study of Obesity. 2004;28(10):1344-8.

18. Larsen MA, Isaksen VT, Moen OS, Wilsgaard L, Remijn M, Paulssen EJ, et al. Leptin to adiponectin ratio - A surrogate biomarker for early detection of metabolic disturbances in obesity. Nutrition, Metabolism and Cardiovascular Diseases. 2018.

19. Li G, Xu L, Zhao Y, Li L, Fu J, Zhang Q, et al. Leptin-adiponectin imbalance as a marker of metabolic syndrome among Chinese children and adolescents: The BCAMS study. PLOS ONE. 2017;12(10):e0186222.

20. Kang DR, Yadav D, Koh S-B, Kim J-Y, Ahn SV. Impact of Serum Leptin to Adiponectin Ratio on Regression of Metabolic Syndrome in High-Risk Individuals: The ARIRANG Study. Yonsei Medical Journal. 2017;58(2):339-46.

21. Magkos F, Fraterrigo G, Yoshino J, Luecking C, Kirbach K, Kelly ShannonC, et al. Effects of Moderate and Subsequent Progressive Weight Loss on Metabolic Function and Adipose Tissue Biology in Humans with Obesity. Cell Metabolism. 2016;23(4):591-601.

22. Clamp LD, Hume DJ, Lambert EV, Kroff J. Enhanced insulin sensitivity in successful, long-term weight loss maintainers compared with matched controls with no weight loss history. Nutrition \&Amp; Diabetes. 2017;7:e282.

23. Nymo S, Coutinho SR, Torgersen L-CH, Bomo OJ, Haugvaldstad I, Truby H, et al. Timeline of changes in adaptive physiological responses, at the level of energy expenditure, with progressive weight loss. 
British Journal of Nutrition. 2018;120(2):141-9.

24. Ryan DH, Yockey SR. Weight Loss and Improvement in Comorbidity: Differences at $5 \%, 10 \%, 15 \%$, and Over. Current Obesity Reports. 2017;6(2):187-94.

25. Nordic Council of Ministers. Nordic nutrition recommendations 2012. Copenhagen: Nordic Council of Ministers,; 2014. Available from: http://norden.diva-

portal.org/smash/get/diva2:704251/FULLTEXT01.pdf.

26. Norwegian Directorate of Health. Dietary Advice to promote Public Health and prevent Chronic Diseases. Oslo: Helsedirektoratet (Norwegian Directorate of Health); 2015 [cited 2015 11/24/15]. 2014: [Available from: https://helsedirektoratet.no/publikasjoner/helsedirektoratets-kostrad.

27. Matsuda M, DeFronzo RA. Insulin sensitivity indices obtained from oral glucose tolerance testing: comparison with the euglycemic insulin clamp. Diabetes Care. 1999;22(9):1462-70.

28. Matthews D, Hosker J, Rudenski A, Naylor B, Treacher D, Turner R. Homeostasis model assessment: insulin resistance and beta-cell function from fasting plasma glucose and insulin concentrations in man. Diabetologica. 1985;28(7):412-9.

29. Isaksen VT, Larsen MA, Goll R, Florholmen JR, Paulssen EJ. Hepatic steatosis, detected by hepatorenal index in ultrasonography, as a predictor of insulin resistance in obese subjects. BMC obesity. 2016;3:39.

30. Bi X, Loo YT, Henry CJ. Does Circulating Leptin Play a Role in Energy Expenditure? Nutrition. 2018.

31. Cohen JC. Chylomicron triglyceride clearance: comparison of three assessment methods. The American journal of clinical nutrition. 1989;49(2):306-13.

32. Lekhal S, Børvik T, Nordøy A, Hansen J-B. Increased Postprandial Triglyceride-Rich Lipoprotein Levels in Elderly Survivors of Myocardial Infarction. Lipids. 2008;43(6):507-15.

33. Alaby Martins Ferreira Y, Claudia Pelissari Kravchychyn A, de Castro Ferreira Vicente S, Munhoz da Silveira Campos R, Tock L, Missae Oyama L, et al. Influence of magnitude of weight loss on Adipo/lep ratio in adolescents with obesity undergoing multicomponent therapy. Cytokine. 2020;131:155111.

34. Talaei M, Nazem F, Ranjbar K. The impact of rapid weight loss (4\%) on leptin, adiponectin, and insulin resistance in elite adult free style wrestlers. The Journal of sports medicine and physical fitness. 2017;57(4):434-40.

35. Elloumi M, Ben Ounis O, Makni E, Van Praagh E, Tabka Z, Lac G. Effect of individualized weight-loss programmes on adiponectin, leptin and resistin levels in obese adolescent boys. Acta Paediatrica. 2009;98(9):1487-93. 
36. Frühbeck G, Catalán V, Rodríguez A, Gómez-Ambrosi J. Adiponectin-leptin ratio: A promising index to estimate adipose tissue dysfunction. Relation with obesity-associated cardiometabolic risk. Adipocyte. 2018;7(1):57-62.

37. Frühbeck G, Catalán V, Rodríguez A, Ramírez B, Becerril S, Salvador J, et al. Adiponectin-leptin Ratio is a Functional Biomarker of Adipose Tissue Inflammation. Nutrients. 2019;11(2):454.

38. Rosenbaum M, Murphy EM, Heymsfield SB, Matthews DE, Leibel RL. Low Dose Leptin Administration Reverses Effects of Sustained Weight-Reduction on Energy Expenditure and Circulating Concentrations of Thyroid Hormones. The Journal of Clinical Endocrinology \& Metabolism. 2002;87(5):2391-4.

39. Rehman K, Akash MSH, Alina Z. Leptin: A new therapeutic target for treatment of diabetes mellitus. 2018;119(7):5016-27.

40. . !!! INVALID CITATION !!! (18).

41. Flier JS, Maratos-Flier E. Leptin's Physiologic Role: Does the Emperor of Energy Balance Have No Clothes? Cell Metabolism. 2017;26(1):24-6.

42. Frithioff-Bøjsøe C, Lund MAV, Lausten-Thomsen U, Hedley PL, Pedersen O, Christiansen M, et al. Leptin, adiponectin, and their ratio as markers of insulin resistance and cardiometabolic risk in childhood obesity. Pediatric Diabetes. 2020;21(2):194-202.

43. Ruhl CE, Harris TB, Ding J, Goodpaster BH, Kanaya AM, Kritchevsky SB, et al. Body mass index and serum leptin concentration independently estimate percentage body fat in older adults. The American journal of clinical nutrition. 2007;85(4):1121-6.

44. Galdas PM, Cheater F, Marshall P. Men and health help-seeking behaviour: literature review. Journal of Advanced Nursing. 2005;49(6):616-23.

\section{Tables}

Table 1: Baseline characteristics of the 28 overweight participants and 17 normal weight controls 


\begin{tabular}{|c|c|c|c|c|}
\hline & $\begin{array}{l}\text { Normal weight } \\
\text { controls }\end{array}$ & $\begin{array}{l}\text { Weight loss } \geq 5 \\
\%\end{array}$ & $\begin{array}{l}\text { Weight loss }<5 \\
\%\end{array}$ & $\begin{array}{l}\text { Sig. } \\
\text { (p) }\end{array}$ \\
\hline $\operatorname{Sex}(M / F)$ & $2 / 15$ & $2 / 11$ & $5 / 10$ & 0.282 \\
\hline Age & $31.0(13)$ & $39.8(13)$ & $36.0(19)$ & $0.254^{\mathrm{M}}$ \\
\hline $\mathrm{BMI}\left(\mathrm{kg} / \mathrm{m}^{2}\right)$ & $21.3(2.2)$ & $33.6(11.5)$ & $39.8(7.5)$ & $0.217^{\mathrm{M}}$ \\
\hline Total body fat (\%) & $26.6(5.9)$ & $50.5(9.5)$ & $49.1(9.1)$ & $0.142^{\mathrm{M}}$ \\
\hline Abdominal fat (\%) & $27.5(6.4)$ & $58.2(7.9)$ & $56.7(3.9)$ & $0.895^{G}$ \\
\hline Gynoid fat (\%) & $36.3(5.8)$ & $54.5(9.1)$ & $53.6(9.8)$ & $0.118^{\mathrm{M}}$ \\
\hline REE (kcal/day) & $1356(185)$ & $1604(441)$ & $1989(465)$ & $0.006^{\mathrm{M}}$ \\
\hline $\begin{array}{l}\text { Systolic blood pressure } \\
(\mathrm{mmHg})\end{array}$ & $105(15)$ & $124(22)$ & $128(15)$ & $0.525^{\mathrm{M}}$ \\
\hline $\begin{array}{l}\text { Diastolic blood pressure } \\
(\mathrm{mmHg})\end{array}$ & $65(10)$ & $80(17)$ & $75(9)$ & $0.208^{G}$ \\
\hline Fasting Glucose (mmol/L) & $4.4(0.7)$ & $5.4(1.3)$ & $5.1(1.0)$ & $0.892^{\mathrm{M}}$ \\
\hline Fasting Insulin ( $\mu \mathrm{mol} / \mathrm{L})$ & $5.5(2.9)$ & $10.8(6.2)$ & $13.2(6.1)$ & $0.142^{\mathrm{M}}$ \\
\hline HOMA-IR & $1.1(0.7)$ & $2.7(1.8)$ & $3.1(1.9)$ & $0.170^{\mathrm{M}}$ \\
\hline WBISI & $147.8(101.5)$ & $55.1(45.6)$ & $55.2(31.2)$ & $0.467^{\mathrm{M}}$ \\
\hline Fasting TG (mmol/L) & $1.0(0.4)$ & $1.2(0.7)$ & $1.5(0.6)$ & 0.011 \\
\hline TGR (mmol/L) & $0.3(0.3)$ & $0.6(0.4)$ & $1.0(0.9)$ & $0.586^{\mathrm{M}}$ \\
\hline TG clearance 6 hrs (\%) & $115.4(62.3)$ & $65.0(96)$ & $61.1(46)$ & $0.413^{\mathrm{M}}$ \\
\hline Leptin (ng/mL) & $8.5(7.4)$ & $32.5(37.0)$ & $41.7(21.8)$ & $0.928^{\mathrm{M}}$ \\
\hline Adiponectin $(\mu \mathrm{g} / \mathrm{mL})$ & $11.8(7.1)$ & $9.0(3.7)$ & $6.8(3.7)$ & $0.525^{\mathrm{M}}$ \\
\hline L:A ratio & $0.6(0,9)$ & $3.8(2.5)$ & $6.1(35.2)$ & $0.683^{\mathrm{M}}$ \\
\hline REE:Leptin ratio & $142.5(134.0)$ & $54.1(51.6)$ & $47.8(51.6)$ & $0.683^{\mathrm{M}}$ \\
\hline Total cholesterol $(\mathrm{mmol} / \mathrm{L})$ & $4.2(0.9)$ & $4.3(1.2)$ & $4.7(1.1)$ & $0.153^{G}$ \\
\hline LDL cholesterol (mmol/L) & $2.6(1.3)$ & $2.7(1.4)$ & $2.9(0.9)$ & 0.107 \\
\hline HDL cholesterol (mmol/L) & $1.6(0.5)$ & $1.3(0.2)$ & $1.1(0.2)$ & 0.004 \\
\hline HDL:LDL ratio & $0.57(0.49)$ & $0.52(0.47)$ & $0.35(0.17)$ & $0.009^{M}$ \\
\hline
\end{tabular}


Baseline anthropometric and metabolic characteristics for all participants. Significance tested between weight loss groups by t-test or Mann-Whitney non-parametric test $\left({ }^{M}\right)$. Parameters without normal variation distribution were transformed to geometric mean $\left({ }^{G}\right)$ if possible before the t-test was performed. Values shown as median (interquartile range). Abbreviations: BMI Body Mass Index. HDL High Density Lipoprotein. HOMA-IR Homeostasis Model Assessment of Insulin Resistance. L:A ratio Leptin:Adiponectin ratio. LDL Low Density Lipoprotein. REE Resting Energy Expenditure. TG Triglyceride. TGR Triglyceride Response. WBISI Whole Body Insulin Sensitivity Index

Table 2: Post-intervention characteristics for the 28 participants 
Weight loss $\geq 5 \%$

$\begin{array}{llllll}\text { Value } & \begin{array}{l}\text { Per cent } \\ \text { change }\end{array} & \begin{array}{l}\text { Sig. } \\ (p)^{\mathrm{W}}\end{array} & \text { Value } & \begin{array}{l}\text { Per cent } \\ \text { change }\end{array} & \begin{array}{l}\text { Sig. } \\ (\mathrm{p})^{\mathrm{W}}\end{array}\end{array}$

Weight $(\mathrm{kg})$

81.1
$(24.1)$

$\mathrm{BMI}(\mathrm{kg} / \mathrm{m} 2)$

30.9

(8.9)

Abdominal fat $(\%)$

51.1

(7.6)

Gynoid fat (\%)

52.0

(11.3)

HOMA-IR

WBISI

$1.4(1.8)$

$-10.0(7.1)$

0.001

119.9

(18.8)

$-8.2(6.2)$

$-7.4(7.6)$

0.001

$-5.4(8.4)$

0.002

92.1

(163.2)

$L: A$ ratio

2.7 (2.2)

REE:Leptin ratio

78.5

(72.9)

REE (kcal/day)

1459

(427)

Fasting TG (mmol/L)

TGR (mmol/L)

$1.1(0.6)$

.51
$(0.59)$

TG clearance 6 hrs (\%)

$75(62.1)$

$-23.1(51.6)$

0.001

39.5

(7.1)

$0.001 \quad 55.6$

(6.5)

-1.1 (4.7)

0.691

51.2

51.2
$(13.5)$

$0.011 \quad 2.6(1.3) \quad-6.8(52.6) \quad 0.807$

$\begin{array}{lll}49.9(133.8) & 0.008 & 67.2\end{array}$

(30.0)

$13.1(50.4) \quad 0.477$

-45.7 (29.9) 0.002

$5.0(4.7)$

$-6.6(26.8)$

0.196

$80.1(92.6) \quad 0.005$

$-8.6(15.6)$

$-9.2(66.0)$

(360)

$-33.6(29.6) \quad 0.327$

Systolic blood pressure

(mmHg)

$121(18) \quad 0.0(8.4)$

$76(20) \quad .6(12.9)$

Diastolic
$(\mathrm{mmHg})$

Fasting Leptin $(\mathrm{ng} / \mathrm{mL})$

$$
\begin{aligned}
& 20.5 \\
& (14.3)
\end{aligned}
$$

$-50.0(36.3) \quad 0.004$

$8.2(4.6)$

Fasting Adiponectin $(\mu \mathrm{g} / \mathrm{mL})$

Fasting Glucose (mmol/L)

Fasting Insulin $(\mu \mathrm{mol} / \mathrm{L})$

LDL cholesterol

$(\mathrm{mmol} / \mathrm{L})$
$8.2(4.6)$

$5.0(1.1) \quad-5.3(11.8)$

$6.3(6.6) \quad-16.6(45.2)$

$2.8(1.0) \quad-11.1(34.9)$

0.006

$8.3(8.4)$

$-4.6(56.6)$

0.470

0.438

4.8 (1.0)

$0.090 \quad 5.1(1.6) \quad-2.1(12.3) \quad 0.339$
71.3
$(68.0)$

0.196

2095
$(360)$

$0.649 \quad 1.3(1.2)$

.67

(1.03)

0.807

66.0
$(42.1)$

$.9(45.8)$

$.0(10.4)$

0.600

0.507

0.754

$-7.1(453.3)$

0.778

0.020

$0.248 \quad 69(16) \quad 1.5(26.4) \quad 0.409$
-24.4
$(148.8)$

$0.074 \quad 120(15) \quad-6.3(15.9)$

$-3.4(15.2) \quad 0.814$

$0.576 \quad 7.1(2.4) \quad 11.0(19.5) \quad 0.433$ 
Post-intervention characteristics and per cent change from baseline characteristics for weight loss ( $\geq 5 \%$ ) and non-weight loss ( $<5 \%)$ groups, respectively. Significance tested between baseline and post-treatment values by Related Samples Wilcoxon Rank Test $\left({ }^{W}\right)$. Values shown as median (interquartile range). Abbreviations: BMI Body Mass Index. HDL High Density Lipoprotein. HOMA-IR Homeostasis Model Assessment of Insulin Resistance. L:A ratio Leptin:Adiponectin ratio. LDL Low Density Lipoprotein. REE Resting Energy Expenditure. TG Triglyceride. TGR Triglyceride Response. WBISI Whole Body Insulin Sensitivity Index

\section{Figures}

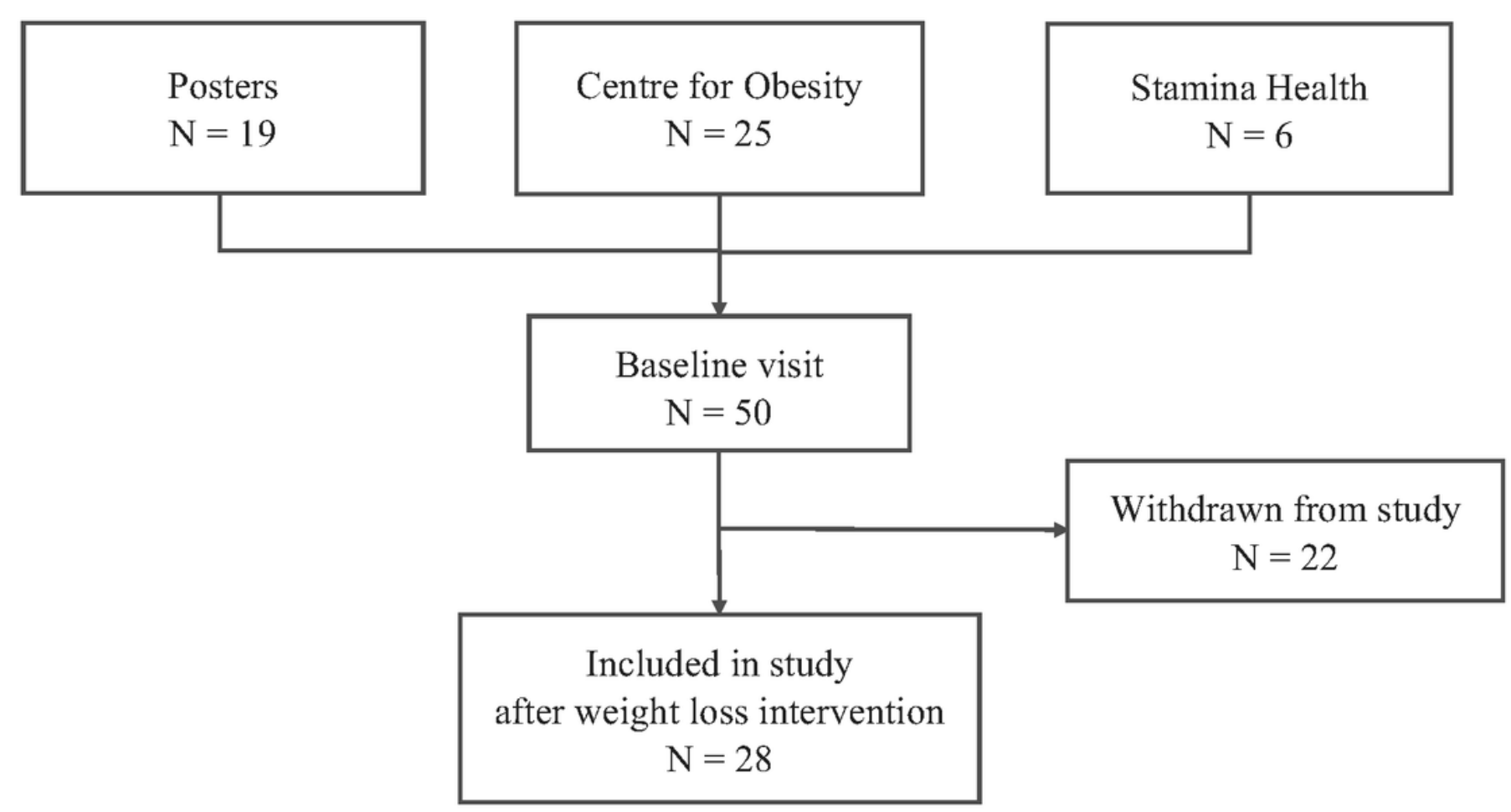

\section{Figure 1}

Flowchart of included participants from posters at UNN, obesity out-patient clinic at UNN and Stamina obesity rehabilitation program, respectively. 
HOMA-IR

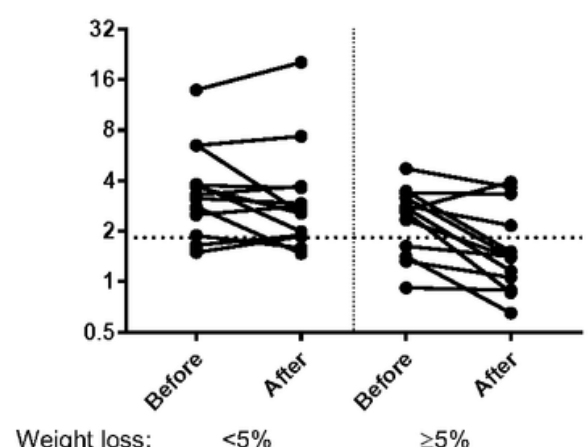

Weight loss:

$<5 \%$

WBISI

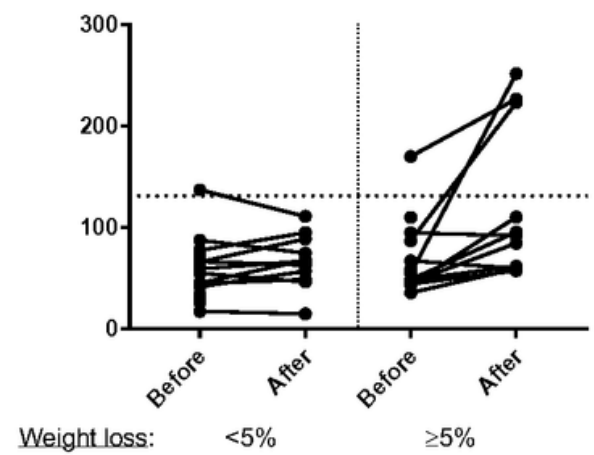

Triglyceride response

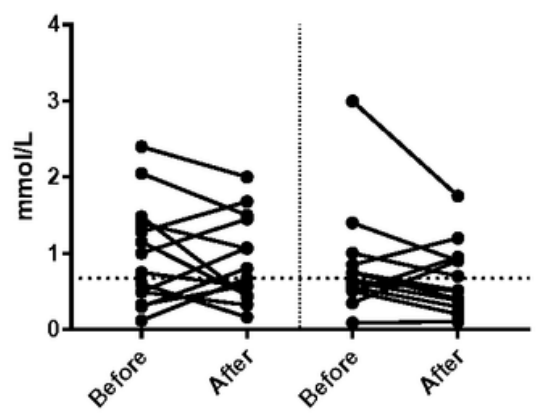

Weight loss: $\quad<5 \%$

TG Clearance at $6 \mathrm{H}$

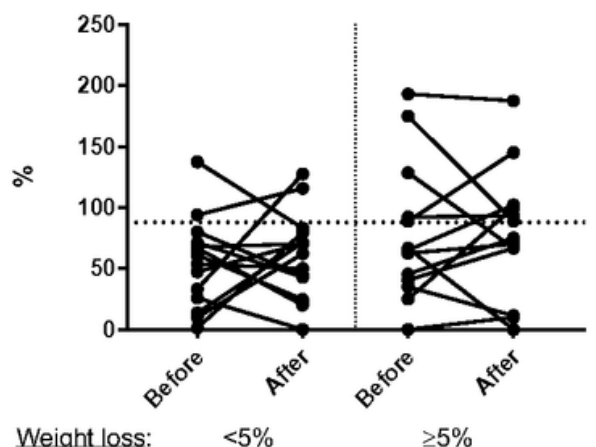

L:A ratio

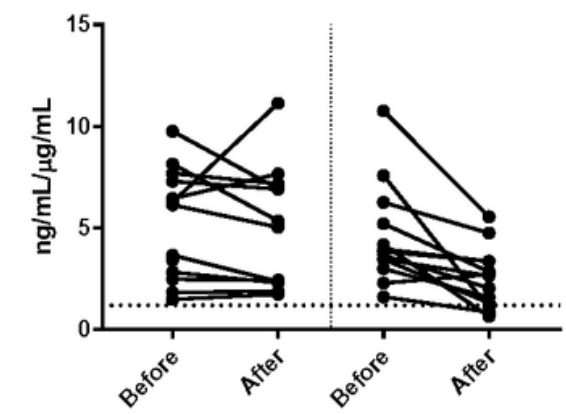

Weight loss:

$\geq 5 \%$
Leptin Sensitivity

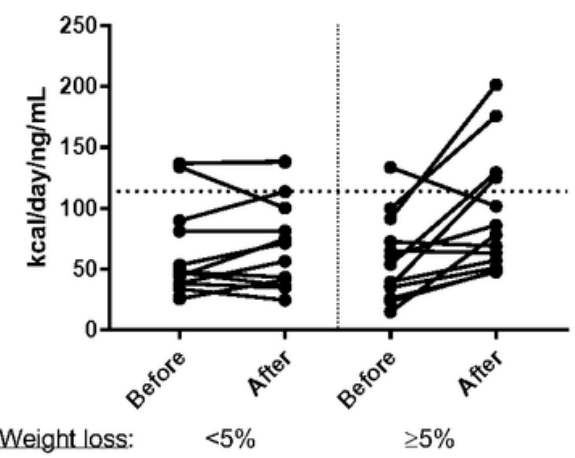

Figure 2

Difference between baseline and post intervention values of A) L:A ratio, B) Indirect leptin sensitivity (REE:Leptin ratio) C) HOMA-IR, D) TG clearance at $6 \mathrm{~h}$ and E) TGR before and after $<5 \%$ and $\geq 5 \%$ weight loss in individual participants. Horizontal lines represent the upper or lower limit of $95 \% \mathrm{Cl}$ in healthy, normal weight controls. 